\title{
1. IMPORTANCE OF NUCLEAR PHYSICS FOR CULTURAL HERITAGE STUDY AND PRESERVATION
}

T he importance of cultural heritage for mankind was once well expressed by the Austrian artist Friedensreich Hundertwasser (1928-2000) when he said: "If we do not honour our past we lose our future. If we destroy our roots we cannot grow." This statement refers almost directly to the two pillars of this review: Investigation and preservation of our cultural treasures. The various contributions summarised in the current review demonstrate that the methods inherent to nuclear physics are capable of following Hundertwasser's vision. The basic concept is to use nuclear radiations of various kinds (X-rays, $y$-rays, electrons, neutrons and ion beams) to analyse the elemental and/or isotopic composition of an object, or to preserve it by irradiation processes.

\subsection{INVESTIGATION OF CULTURAL HERITAGE OBJECTS}

It is clear that precious cultural heritage objects should remain unaltered after they are exposed to analytical investigation. Therefore non-destructive methods are of crucial importance for investigations. This simply means that the (unavoidable) side-effects of an irradiation must not be noticeable on the object of interest, now or in the future.

This can be primarily achieved by reducing the intensity of the irradiation to very low levels. In order to obtain meaningful analytical information, the low primary irradiation has to be balanced by a correspondingly high detection efficiency of the secondary signal one wants to analyse. Great strides in this direction have been undertaking in recent times, opening up many possibilities to analyse valuable pieces of art.

\subsubsection{ION BEAMS}

Although ion beam analysis developed later than other methods - simply because suitable accelerators only became available in the second half of the $20^{\text {th }}$ century, it is now the most versatile technique for investigating objects of cultural significance. This is due to the flexibility of ion beams, where the beam species (protons, alphas, heavy ions), the energy, the intensity, and the diameter of the beam (sub-millimeter to sub-micron size) can be varied in a suitable way. In addition, the efficiency and resolution of detector systems for X-rays, $y$-rays, and charged particles have greatly improved over the years. An important aspect for ion beam analyses of art objects is the use of an external beam, because often these objects cannot be brought inside the accelerator vacuum system (as an example, see the cover picture of this report). A multitude of different ion beam techniques is now available: NRA (Nuclear Reaction Analysis), PIXE (Proton Induced X-Ray Emission), PIGE (Proton Induced Y-ray Emission), RBS (Rutherford Back-Scattering), ERDA (Elastic Recoil Detection Analysis). All of these are discussed in this review.

\subsubsection{X-RAY AND $Y$-RAY BEAMS}

Since the birth of nuclear physics around 1900, X-rays have been available from the bremsstrahlung radiation emitted by energetic electrons as they pass through materials, and from $X$-rays emitted when an electron vacancy is filled in an atomic orbit (characteristic $X$-rays). The most common method for cultural heritage investigation is XRF (X-ray Fluorescence). Due to its different depth-sensitivity it is complementary to PIXE, and is sometimes combined with it. Portable instruments make XRF a very valuable method for studying objects which cannot be moved to an accelerator. The invention of polycapillary focusing lenses for X-rays led to the development of Micro-XRF, which improved the spatial resolution and thus the versatility of analysing distributions of trace elements. Such developments are being further advanced by utilising the very powerful $X$-rays from electron synchrotrons which are pushing Micro-XRF into the nanometer spatial regime. High-energy $\mathrm{X}$-rays from free-electron laser facilities will likely add another dimension to the quest for ever more detailed X-ray studies of culture heritage objects. 
The European initiative for Extreme Light Infrastructure (ELI) laboratories at ELI-NP in Romania, will provide tunable $\mathrm{y}$-rays from inverse Compton scattering of laser light on a high-energy electron beam. This will allow Nuclear Resonance Fluorescence (NRF) studies of isotope-specific trace element distributions to be performed with unprecedented sensitivity.

\subsubsection{NEUTRON ACTIVATION ANALYSIS (NAA)}

Shortly after the neutron was discovered by Chadwick in 1932, Fermi and others started to convert stable isotopes of many elements into radioactive ones by neutron absorption. In 1936, Hevesy and Levi in Copenhagen realised the analytic power this method had to measure trace elements (particularly REE = Rare Earth Elements). To this day, NAA is used at research reactors, where highintensity neutron sources are available. In combination with high-resolution Ge detectors complex y-spectra from irradiated material can be disentangled, allowing the simultaneous measurement of the concentrations of up to 30 trace elements. Although NAA usually requires bringing the cultural heritage object (or a representative sample of it) to the reactor for neutron irradiation, chemical pre-treatment of the material is not necessary, preserving the original composition of the object. NAA turns out to be particularly useful in the study of trace element distributions in ceramics, helping to determine questions of provenance.

\subsubsection{NUCLEAR MAGNETIC RESONANCE (NMR)}

A frequently applied NMR method in medical diagnosis is called MRI (Magnetic Resonance Imaging), which allows details of soft tissue in humans to be studied by resonantly exciting the nuclear spin of hydrogen in a strong magnetic field. Since the excitation happens with radiofrequency radiation, only non-ionising radiation is used. $A$ big step towards using NMR for cultural heritage was the development of a portable NMR instrument called NMRMOUSE (Mobile Universal Surface Explorer).

\subsubsection{AGE DETERMINATION}

If the absolute age of an object containing organic carbon is of interest, ${ }^{14} \mathrm{C}$ dating is often used. Since this requires taking a small piece of material from the object, it is not a truly non-destructive method. However, counting ${ }^{14} \mathrm{C}$ atoms directly by accelerator mass spectrometry (AMS), rather than counting the infrequent $\beta$-decays (the original method), has increased the detection efficiency of ${ }^{14} \mathrm{C}$ by a factor of about $10^{6}$. This then allows ${ }^{14} \mathrm{C}$ measurements to be performed on very small samples, sometimes as low as a few micrograms of carbon, with negligible effects on the sampled object. The age range extends back to some ten half-lives of ${ }^{14} \mathrm{C}$, i.e. to about 50,000 years. The determination of an absolute age from the measured ${ }^{14} \mathrm{C}$ content requires a calibration curve, which is updated about every five years by an international collaboration committee.

An unusual help to uncover recent art forgery comes from the so-called ${ }^{14} \mathrm{C}$ bombpeak, an abrupt doubling of the atmospheric ${ }^{14} \mathrm{C}$ content around 1960 due to the intense atmospheric nuclear weapons testing period. Finding this ${ }^{14} \mathrm{C}$ excess in an object of supposedly prenuclear origin is an unambiguous proof of forgery.

Inorganic materials, such as ceramics, can be subjected to luminescence dating. Thermo-Luminescence (TL) and more recently Optically Stimulated Luminescence (OSL) are being used, preferably on selected quartz grains from the object to be dated. Here the age determination depends on the production of luminescence centres in a mineral through the radiation dose received from internal and environmental radioactivity. The latter is sometimes difficult to reliably assess for the whole time period to be dated, resulting in a somewhat lower precision than ${ }^{14} \mathrm{C}$ dating. On the other hand, the age range of $\mathrm{TL}$ and OSL is about 300,000 years, considerably longer than that of ${ }^{14} \mathrm{C}$.

\subsection{PRESERVATION OF CULTURAL HERITAGE OBJECTS}

Preservation often requires high intensities of irradiation which may induce changes in the object of interest. One of the main applications is the sterilisation of an object by $\mathrm{Y}$-rays, a method widely used for medical equipment, and sometimes for food as well. The purpose of the irradiation is to kill any bioactivity (e.g. bacteria, fungi, woodworms), which could have adverse effects on the conservation of an object. However, since finite effects are expected on the irradiated objects due to using a high dose of ionising radiation, a careful assessment of these effects must be performed prior to any preservation procedure. Another radiation-assisted procedure for the preservation of objects is consolidation by radiopolymerisation of suitable material added to the object. It is clear that in the various preservation procedures the benefit of a prolonged conservation must be weighed against the unavoidable side effects on the objects one wants to preserve.

\subsection{PRESERVE THE OLD, BUT KNOW THE NEW}

This well-known Chinese proverb can be a guideline for the importance of cultural heritage investigations and preservations - just like Hundertwasser's saying discussed earlier. This review paper demonstrates that we are well on the way to following these guidelines. It is gratifying that nuclear physics, which the public often connects only with the threat from nuclear weapons, radioactivity 
and disasters at nuclear power plants, can contribute in such a significant way to a deeper understanding of our cultural heritage.

There are countless objects of great value stored in museums around the world. The nuclear physics methods described in this review, as well as some other natural science methods, can be used to gain a deeper understanding of their cultural significance. Many of these objects are unique witnesses to the past, and should be investigated with the utmost care. Since one can expect a steady improvement in analytical methods in the future, the value of cultural heritage objects will increase. Therefore, preservation without alteration is a very important goal. In a way, the treatment of material from the moon brought back by the Apollo astronauts in the early 1970 s can be a model. Some of this material is stored away for future generations when improved analyses will be able to extract more information from it than is currently possible.

In general it seems likely that the desire to understand our cultural heritage will grow. This is based on the assumption that from more detailed studies of the past we will simply learn more about us, i.e. the human species which inhabits the Earth now. Besides the methods described in the current review, there are many other ways to enlarge our understanding of cultural heritage, both by methods of sciences and the humanities. Among them a very promising complementary technique is the rapidly evolving field of ancient DNA studies, which will undoubtedly make a major contribution to a better overall understanding of our cultural heritage - and ourselves as well.

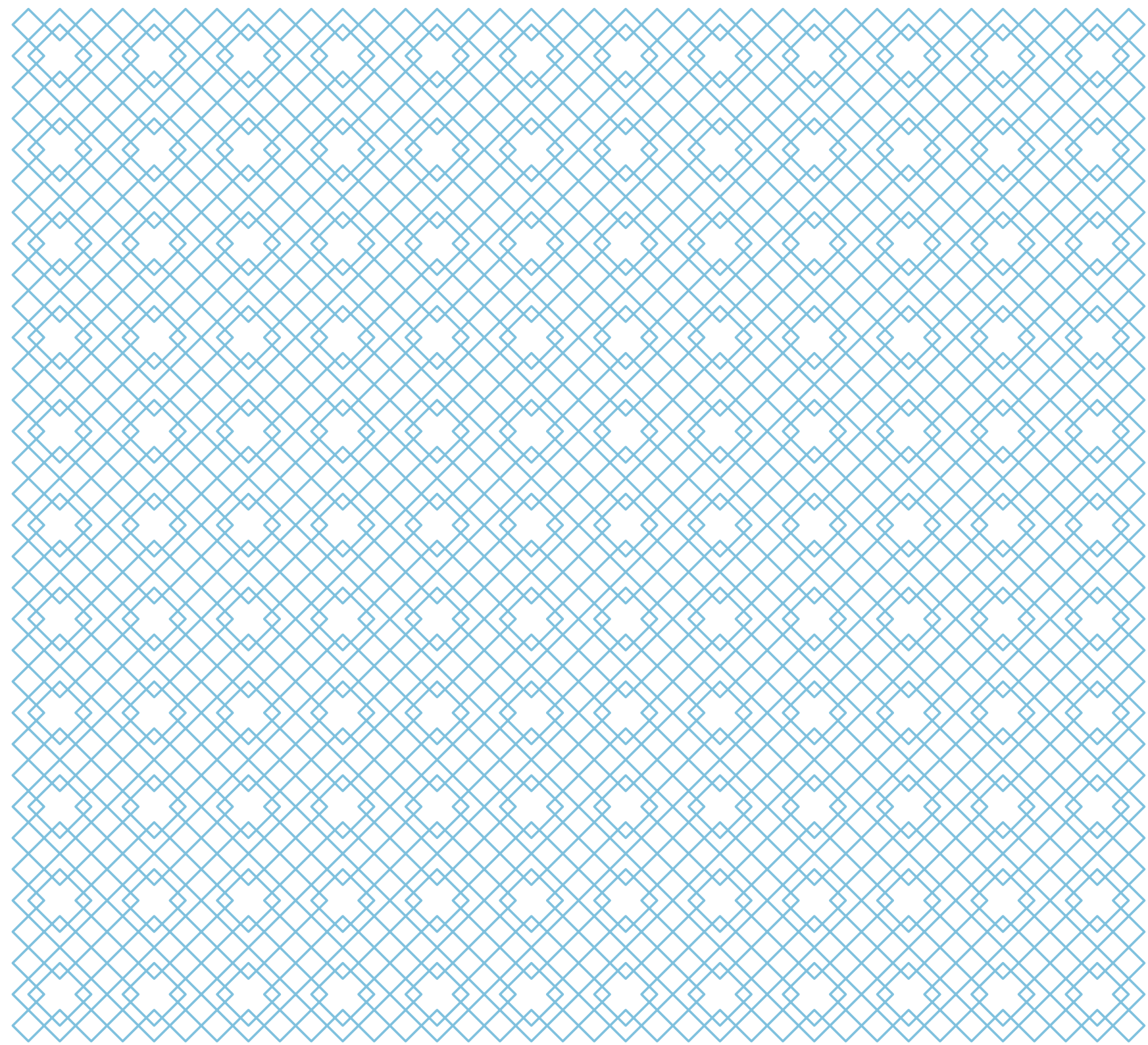

